\title{
Führen mit Zielen in der Automobilindustrie - wie evidenzbasiert ist die Umsetzung in der Praxis?
}

\author{
Annika Burkhardt-Waldherr' ${ }^{1}$ Simone Kauffeld'
}

Online publiziert: 27. April 2020

( ) Der/die Autor(en) 2020

\section{Zusammenfassung}

Dieser Beitrag in der Zeitschrift „Gruppe. Interaktion. Organisation (GIO)“ stellt eine qualitative Studie zum Umsetzungsstands des Führen mit Zielen in einem Beispielunternehmen dar und beleuchtet, welche wissenschaftliche Erkenntnisse den Weg in die Praxis gefunden haben. Führen mit Zielen ist ein altbewährtes Mittel zur Motivation und Steigerung der Leistungsfähigkeit in Organisationen. Obwohl schon viel geforscht wurde zu Zielsetzungen und deren motivierenden Wirkungen, sind in der Praxis kontraproduktive Varianten anzutreffen. Hieraus leitet sich die Frage ab, welche Evidenzen zum Führen mit Zielen in der Praxis bekannt sind und welche tatsächlich Anwendung finden. In einem qualitativen Vorgehen wurde dies untersucht, indem eine Exploration über das vorherrschende Wissen und dessen Umsetzung in Form von Interviews mit Vertretern eines Automobilunternehmens vorgenommen wurde. Es kann festgestellt werden, dass das Verständnis über das Führen mit Zielen im Unternehmen nicht durchgängig mit dem in der Wissenschaft beschriebenen Wissen übereinstimmt. Die Interviewergebnisse zeigen, dass insbesondere der moderierende Effekt der Zielbindung wenig beachtet wird und stattdessen ein sehr hoher Fokus auf den Zielplanungsprozess gelegt wird. Ziele werden überwiegend vorgegeben anstatt vereinbart und nur zu sehr geringem Maße transparent kommuniziert. Oft sind sie in Form von Maßnahmen oder Aufgaben beschrieben und werden nicht explizit als Ziel wahrgenommen. Der Einsatz von einem kontinuierlichen Feedback ist bereits weitflächig etabliert und wird oft durch visuelle Elemente (z. B. Steuerungstafeln) unterstützt. Dieser Beitrag erläutert, wie durch die Beachtung von wissenschaftlichen Erkenntnissen eine höhere Wirksamkeit der Zielsysteme erreicht werden kann.

Schlüsselwörter Evidenzbasiertes Management $\cdot$ Führen mit Zielen $\cdot$ Führung $\cdot$ Kennzahlen · Management by Objectives $\cdot$ Zielsetzung

Dipl. Psych. Annika Burkhardt-Waldherr

a.waldherr@tu-braunschweig.de

Prof. Dr. Simone Kauffeld

s.kauffeld@tu-braunschweig.de

1 Institut für Psychologie, Lehrstuhl für Arbeits-,

Organisations- und Sozialpsychologie, Technische Universität

Braunschweig, Braunschweig, Deutschland 


\title{
Goal setting within the automotive industry-how evidence-based is the transfer into practice?
}

\begin{abstract}
This journal article in the magazine "Gruppe. Interaktion. Organisation (GIO)" represents a qualitative study regarding the implementation status of goal setting as part of leadership within a company and highlights which scientific findings have found their way into practice. Goal setting is a well-established and tested instrument to increase motivation and performance in organizations. Although, a big amount of research and proven evidence on the motivational effect of goal setting is published, modifications with reverse effects can be found in practice. By this, the following question derives: Which evidence is known and how it is used in practice? This will be analyzed in-depth in a qualitative way by exploring the prevailing knowledge and its implementation by conducting interviews with representatives of an automotive company. The results show that the understanding of goal setting in the company does not consistently correspond to the knowledge described in science. The results show that the effect of goal commitment is not taken seriously into account, but instead a very high focus lays on the goal planning process. Goals are mostly given rather than commonly agreed and they are communicated to a very limited extent transparently. They are often described as measures or tasks and therefore not explicitly perceived as goals. However, the use of continuous feedback is already more common and is often supported by visual elements (e.g. control panels). This article explains how a corporate goal system can be more effective considering scientific evidence.
\end{abstract}

Keywords Evidence-based management $\cdot$ Goal setting $\cdot$ Key performance indicator (KPI) $\cdot$ Leadership $\cdot$ Leading with goals $\cdot$ Management by objectives

\section{Das Aufleben eines altbewährten Ansatzes}

Das Führen mit Zielen hat sich in der organisationalen Praxis etabliert. Bereits $60 \%$ der DAX Unternehmen haben dies in ihren Führungsalltag integriert sowie in verschiedenen Kennzahlensystemen verankert (Schwaab 2010). Der Einsatz von Zielen in der Führungsaufgabe ist nicht neu, sondern hat im industriellen Umfeld eine lange Tradition (Bungard und Kohnke 2002). Die Grundlage wurde in den Zeiten des Taylorismus und des Scientific Management (Taylor 1911) gelegt, welche die Ausrichtung nach Zielen zur wirtschaftlichen Ergebnissicherung in der industriellen Produktion geprägt haben (Breisig 2001). Durch Zielsetzungen hat die Führungskraft als ,wissender Vorarbeiter“ den Mitarbeitern die an sie gestellte Aufgaben in kleine Teilziele zerlegt. Hierauf aufbauend entstand der Management by Objectives Ansatz, der in den Arbeiten von Drucker (1954) erstmals systematisch beschrieben worden. Der Ansatz diente zur klaren Strukturierung von Führung in den Unternehmen. Ziele wurden über mehrere Führungsebenen im Unternehmen kaskadiert und Führungskräfte wurden an deren Erfüllung gemessen. Drucker schaffte hiermit einen bis heute oft verwendeten Führungsansatz. In den Zeiten der betrieblichen Demokratisierung und Mitbestimmung der 70er Jahren wurde der Wunsch nach Beteiligung der Mitarbeiter laut. Ziele sollten nicht nur zum Aufgabenbefehl, sondern auch zur Delegation von Verantwortung an die Mitarbeiter genutzt werden. So fanden psychologische Ansätze den Einzug in unternehmerische Zielsysteme. Hierzu gehören Ansätze zur Leistungs- und Hand- lungsmotivation (z. B. Heckhausen 1977; McClelland et al. 1953), die im Rahmen der Zielsetzungstheorie von Locke und Latham (1990) berücksichtigt wurden. Die Kombination von wirtschaftlichen und psychologischen Ansätzen traf den Nerv der Zeit für eine klare Orientierung und eine Stärkung der Motivation in Unternehmen. Hierdurch sind die noch heute anzutreffenden Zielvereinbarungen in Unternehmen entstanden. Sie stellen einen Aspekt der transaktionalen Führung dar, bei der für eine gezeigte Arbeitsleistung (Zielerfüllung) eine Gegenleistung (Bonus) erfolgt (Burns 1978). In der Führungsforschung wird diesem Ansatz vorgeworfen den Fokus zu sehr auf die extrinsische Motivation zur Zielerfüllung zu legen (Felfe 2015). Aktuell erlebt dieser Klassiker jedoch eine Renaissance in der Praxis (Voss 2015).

Unternehmen unterliegen einem Transformationsprozess, indem eine Neuausrichtung gefordert wird und damit der Bedarf nach Orientierung steigt. Das Umsetzungstempo dieser Veränderung ist ein entscheidender Faktor, wodurch neue Formen der Zusammenarbeit im Unternehmen entstehen und der Ruf nach Innovationen lauter wird. Um schnellere Entwicklungen zu unterstützen, sind funktionsübergreifende Teamzusammensetzungen, verstärkt flache Hierarchien und damit verbunden größere Führungsspannen notwendig (Hay Group 2011; Sackmann 2017). Der Einsatz von Zielen in der Führungsarbeit soll die Kommunikation von strategischen Entscheidungen sowie die Vermittlung von dessen Konsequenzen für die Arbeit unterstützen (Domke et al. 2019). Durch eine klare Zielorientierung können Energien und Ressourcen stärker gebündelt sowie fokussiert eingesetzt werden. Ziele sind zukünftig nicht 
Abb. 1 Modell der Zielsetzungstheorie nach Locke und Latham (1990) inklusive Moderator- und Mediatorvariablen. (Eigene Darstellung)

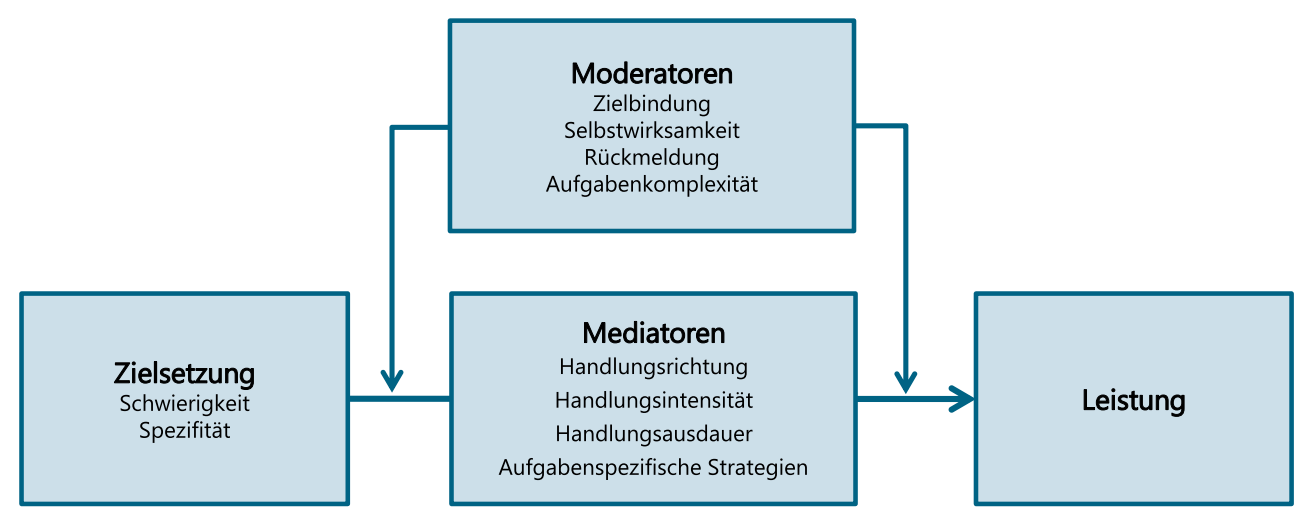

2 Die Zielsetzungstheorie

mehr ein Kontrollinstrument der Führungskraft, sondern sollen den ursprünglichen Gedanken zum Befähigen der Mitarbeiter fördern (Voss 2015). Durch eine Ziel- und Aufgabenklarheit kann die Befähigung der Mitarbeiter gestärkt werden und Führungskräfte erhalten Freiräume für strategische Tätigkeiten. Somit gewinnt der Einsatz von Zielen eine neue Bedeutung. Neben den veränderten Rahmenbedingungen gibt es auch kritische Stimmen, dass psychologische Faktoren in den aktuell angewendeten Zielsystemen nicht mehr angemessen beachtet werden (Bungard und Kohnke 2002). Demnach haben betriebswirtschaftliche Faktoren, wie Kontroll- und Planungsmechanismen (Drucker 2007), eine größere Bedeutung in aktuellen Zielsystemen als die Befähigung der Mitarbeiter zum eigenverantwortlichen Handeln. So ist es nicht verwunderlich, dass vielen Zielsystemen ein zu starker Kontrollfokus nachgesagt wird, anstatt die angestrebte motivierende Wirkung eines Personalführungsinstruments (Voss 2015). Zielvereinbarungen werden in Befragungen immer mehr als eine Ursache für Leistungsdruck und Arbeitsintensivierung in Organisationen empfunden (Hoppe und Rau 2017). Im Kontext der Gesundheitsförderung und der Erhaltung der Arbeitsfähigkeit kann dies als ein wichtiger Faktor in der Arbeitsgestaltung angesehen werden, der einer Überarbeitung bedarf (Franke et al. 2015). Die veränderten Rahmenbedingungen in Unternehmen und die damit verbundenen Anforderungen an das Führen mit Zielen geben Anlass, die Anwendung in der Praxis nach heutigem Wissensstand einer Untersuchung zu unterziehen. Hierbei sollen die tatsächliche Umsetzung des Führens mit Zielen sowie die Beachtung von vorhandenen Evidenzen der Zielsetzungstheorie betrachtet werden. Es wird auf den Einsatz von Zielen in der unternehmerischen Praxis anhand qualitativer Interviews eingegangen. Abgeschlossen wird der Artikel mit der Erkenntnisgewinnung in Form von Impulsen für Forschung und Praxis sowie einer kritischen Reflexion des Vorgehens.
Ziele zu erreichen ist ein zentrales Element von Führung in Organisationen. Der Einsatz von Zielen dient je nach Betrachtungsperspektive unterschiedlichen Zwecken (Putz und Lehner 2002). So dient es Planungszwecken, wie zum Beispiel welche Ressourcen für welche Aufgabe eingeplant werden, oder auch Berichtszwecken, um Erfolgsbeiträge messbar zu machen (Drucker 2007). Aber es unterstützt auch Motivationszwecke, wie dass Organisationsmitglieder Anreize, Orientierung oder Sinn zum Handeln erhalten (Locke und Latham 2013). Hinter dem letztgenannten Zweck steht die psychologische Grundannahme, dass Ziele Menschen zum Handeln motivieren und somit positiv das Leistungsverhalten beeinflussen (Hacker 1999). Die psychologischen Wirkzusammenhänge zwischen Zielen und der Arbeitsleistung wurden in der Zielsetzungstheorie beschrieben (Locke und Latham 1990, 2006). Demnach sollen insbesondere spezifische und schwierige Ziele zu einer hohen Leistung führen. Die Theorie betrachtet nicht nur die Zielsetzung, sondern auch weitere psychologische Wirkmechanismen, die durch die Zielsetzung ausgelöst werden und diese beeinflussen (siehe Abb. 1). Hierbei wird von sogenannten Mediator- und Moderatorvariablen gesprochen.

Unter Mediatoren werden Variablen verstanden, die zwischen der Zielsetzung und dem angestrebten Leistungsverhalten in der Zielbearbeitung vermitteln. Diese Variablen beziehen sich auf die Zieleigenschaften und deren Beschaffenheit. Hinsichtlich der Beschaffenheit von Zielen ist grundsätzlich anzunehmen, dass von der Spezifität der Ziele auch eigenständige handlungsfördernde Wirkungen ausgehen (Bayer et al. 2010). Demnach umfasst das Ziel Informationen zur Handlungsrichtung, -ausdauer sowie -intensität. Anhand dieser Informationen kann ein Abgleich mit bisherigen aufgabenspezifischen Strategien stattfinden. Ein Ergebnis dieses Abgleichs kann sein, dass auf vorhandene Strategien zurückgegriffen wird oder dass neue Strategien aufgebaut werden müssen. Die Informationen helfen entsprechende Handlungen oder Vorgehensweisen auszuwäh- 
len, die für den Zielempfänger die höchste Erfolgswahrscheinlichkeit darstellen.

Darüber hinaus gibt es Moderatoren des Zielprozesses, die in der Zielbearbeitung wirken. Sie sind für das Ausmaß und die Art des Zusammenhangs zwischen der Zielsetzung und der Zielerreichung verantwortlich. Hierzu gehört eine kontinuierliche Rückmeldung zum Zielerreichungsstand in Form von Feedback. Die Kombination von Zielen mit Leistungsrückmeldungen hat einen höheren Effekt auf die tatsächlich gezeigte Leistung als Ziele ohne Rückmeldungen (Neubert 1998). Die Überlegenheit der Kombination beider Größen wird damit erklärt, dass Ziele und Rückmeldungen zwei sich ergänzende Funktionen in Bezug auf das Leistungshandeln erfüllen. ,Ziele organisieren und lenken Handlungen, während Rückmeldungen eine Kontrolle des Fortschritts zum Ziel erlauben“" (Wegge 2015, S. 183). Ein weiterer Moderator ist die Zielbindung, die ausdrückt, ob das zu erreichende Ziel für die Person wichtig ist. Je wichtiger das Ziel für die Person ist, umso mehr wird sie sich dauerhaft anstrengen das Ziel zu erreichen (Klein et al. 2012). Ziele, bei denen eine geringe Zielbindung besteht, werden schneller aufgegeben als diejenigen mit hoher Bindung (Brandtstädter 2015). Diese Erkenntnis ist besonders beim Setzen von langfristigen Zielen zu beachten. In der Theorie wird weiterhin die Selbstwirksamkeitsüberzeugung genannt. Hierunter wird die Überzeugung einer Person verstanden, an sie gerichtete Aufgaben erfüllen zu können (Bandura 2012). Befunde zeigen, dass Personen mit einer geringen Ausprägung der Selbstwirksamkeit kein schwieriges Ziel wählen oder sich zu ihm bekennen, anders bei einer hoch ausgeprägten Selbstwirksamkeit (Latham und Locke 2007). In der Zielbearbeitung zeigt es sich darin, ob die Person sich zutraut das Ziel selbstständig umzusetzen und auf bisherige Umsetzungsstrategien vertraut. Hieran knüpft die letzte Moderatorvariable in diesem Modell an. Meistens sind Ziele in zu erledigende Arbeitsaufgaben integriert. Somit ist neben den Zieleigenschaften auch entscheidend, wie komplex die zu erledigende Aufgabe ist. Dies wird als Aufgabenkomplexität bezeichnet. Bei einfachen Aufgaben ist der leistungsförderliche Einfluss von herausfordernden, spezifischen Zielen stärker ausgeprägt als bei komplexen Aufgaben (Locke und Latham 2002).

Die abhängige Variable in dem Modell ist die Leistung, welche das durch das Ziel bezweckte Handlungsresultat darstellt. In Bezug auf die Arbeitsleistung ist dies in unterschiedlichen Ergebnisformen zu messen, wie zum Beispiel fehlerfreies Arbeiten, erfolgreiche Projektabschlüsse, Innovationen.

Das Besondere ist, dass die Kernaussagen der Theorie nicht nur im Labor nachgewiesen sind, sondern auch in zahlreichen Felduntersuchungen und Längsschnittstudien wiederholt bestätigt wurden (Kauffeld und Schermuly 2019; Locke und Latham 2013; Wegge 2015).

\section{Einsatz von Zielen in der betrieblichen Praxis und deren Tücken}

Trotz klarer Befunde der Zielsetzungstheorie, kann die Umsetzung in der Praxis misslingen. Die erfolgreiche Anwendung von Zielsetzungen in der Praxis wird als „eigene Kunst" beschrieben (z.B. Wegge 2015). Dabei ist sowohl die Anwendung als auch die antizipierte Wirkung zu betrachten.

In der Anwendung sind insbesondere im komplexen Gefüge von Organisationen einige Fallstricke zu finden. So ist in vielen Unternehmen zu beobachten, dass jeder Bereich seine eigenen Ziele formuliert. Ein Abstimmen mit der Gesamtstrategie erfolgt selten und ein Silo-Denken kann sich hierdurch ausprägen (Schwaab 2010). Eine andere häufiger anzutreffende Tücke ist, dass Gruppen- oder Organisationsziele gesetzt werden, die kollektiv bearbeitet werden sollen, jedoch nur von einer einzelnen Person umsetzbar sind und in dem Sinne Individualziele darstellen (Hoch et al. 2009). Ein Beispiel in der Automobilbranche ist die Investitionsquote auszuschöpfen, wobei die Entscheidung über Investitionen nur durch eine Person getroffen werden kann. Somit bleibt die motivierende Wirkung auf die Gruppe aus. Darüber hinaus sind in der Praxis anstatt Zielvereinbarungen überwiegend Zielvorgaben anzutreffen (Bungard und Kohnke 2002). Generell wird in vielen wissenschaftlichen Ausführungen der Vereinbarungscharakter empfohlen, auch wenn die motivierende Wirkung nicht als stärker nachgewiesen gilt als bei Zielvorgaben, die vom Zielempfänger akzeptiert werden (Barends et al. 2016). Jedoch zeigen Untersuchungen, dass in der längeren Zusammenarbeit von Teams oder Mitarbeiter und Führungskraft Zielvereinbarungen den Zielvorgaben überlegener sind (Haslam et al. 2009). Die Überlegenheit zeigt sich im Zeithorizont der Zielsetzung. Bei kurzfristig zu erreichenden Zielen können Zielvorgaben vorgezogen werden, da die Zielsetzung durch die Führungskraft entschieden wird und dies weniger Zeit erfordert als partizipative Entscheidungsprozesse. Bei langfristigen Zielen wird die Zielvereinbarung empfohlen, da die Mitarbeiter hierdurch stärker Selbstführungskompetenzen ausbilden und die Führungskraft weniger planerische Tätigkeiten übernehmen muss (Nerdinger 2008). Wichtig ist, dass auch bei Zielvorgaben der Sinn des Ziels erklärt wird und somit auch eine Bindung zu dem Ziel aufgebaut wird. In diesem Zusammenhang wird vom „Tell-and-Sell“ Mechanismus gesprochen (Latham et al. 1988). So sollte den Mitarbeitern das gesetzte Ziel erklärt (Tell) und auch die Wichtigkeit der Zielerreichung für die Organisation herausgestellt werden $(\mathrm{Sell})$. Wenn diesem Mechanismus gefolgt wird, können Zielvorgaben genauso motivierend sein wie Zielvereinbarungen. In diesem Zusammenhang wird auch von einer konstruktiven Passung zwischen der Vision (Warum?) und den Zielsetzungen (Was?) gesprochen (Ber- 
son et al. 2015). Die Vision stellt hierbei die Wichtigkeit der Zielerreichung für die Organisation dar, also warum bestimmte Ziele zu erreichen sind. Studien zeigen, dass vielen Beschäftigten die wesentlichen Unternehmensziele nicht bekannt sind und damit die Verbindung zur eigenen Leistung nicht erfolgen kann (Covey 2018). Zudem sind viele Ziele durch Kennzahlen operationalisiert. Heutzutage sollen vermehrt Prognosen basierend auf diesen Kenngrößen abgeleitet sowie Erfolgsbeiträge messbar gemacht werden. Der Einsatz von sogenannten Dashboards, in denen einen Vielzahl von Kennzahlen übersichtlich für das Management dargestellt wird, ist ein großer Trend in der betrieblichen Praxis (Neuner und Neuner 2014). Obwohl in Unternehmen Kennzahlen im Zielkontext kontinuierlich verfolgt werden, zeigen Studien, dass die erhobenen Fakten selten evidenzbasiert ausgewertet oder zur Entscheidungsfindung herangezogen werden (Posch und Speckbacher 2012; Rousseau 2006). Demnach werden Entscheidungen trotz des hohen Aufwandes für die Erstellung von Berichten oft anhand des Bauchgefühls der Entscheidungsträger getroffen (Pfeffer und Sutton 2007).

Obwohl dem Einsatz von Zielen positive Wirkungen zugesprochen werden, wird in der Praxis auch von dysfunktionalen Effekten berichtet (Bungard und Kohnke 2002). Demnach sind Zielvereinbarungen eine Quelle für Zeit- sowie Leistungsdruck und können negativ auf das gesundheitliche Wohlbefinden der Mitarbeiter wirken (Hoppe und Rau 2017). Auch eine kontinuierliche Zielerschwerung kann demotivierend sein. Es ist zwar ein Anstieg der Leistung bei schwierigen Zielen zu beobachten, jedoch kann dies in ein individuelles Leistungsplateau münden und sich dann in einer Überforderung ausdrücken (Wegge 2015). Zusätzlich werden mehr kritische Stimmen zu unerwünschten „Nebenwirkungen" laut, wie zum Beispiel die Zunahme der Risikobereitschaft oder unethisches Handeln bei schwierigen Zielen (Wegge 2015). Diese Nebenwirkungen lassen sich auf die eingangs erwähnte Fokussierung auf die extrinsische Motivation beziehen, demnach die angestrebte Belohnung bei der Zielerreichung maßgeblich für das Handeln ist. Im Gegensatz hierzu ist die intrinsische Motivation auf die Zielerreichung ausgelegt und nicht auf die damit verbundene Belohnung (Böhnisch et al. 2000). Die intrinsische Motivation ist bei selbstgesetzten und auch vereinbarten Zielen stärker ausgeprägt. Darüber hinaus kann es wichtig sein, sich von Zielen zu lösen oder diese anzupassen, wenn sich z.B. Rahmenbedingungen geändert haben (Brandtstädter 2015; Kauffeld und Schermuly 2019).

\section{Eine Interviewstudie zum evidenzbasierten Umgang mit Zielsetzungen}

Die Anwendung von wissenschaftlichen Erkenntnissen in der Praxis wird im Forschungsrahmen des evidenzbasierten Managements beleuchtet (Biemann et al. 2012; Kauffeld 2019). Der Ansatz des evidenzbasierten Managements folgt den Prinzipien der evidenzbasierten Medizin mit dem Ziel Entscheidungen in Organisationen auf bestmöglicher wissenschaftlicher Evidenz zu treffen, analog der Evidenz zur Wirkung eines Medikaments (Rousseau 2006). Hierbei ist nicht nur die Frage, was die nützlichsten Informationen sind, sondern auch wie diese anwendungs- und kontextbezogen genutzt werden können. In diesem Zusammenhang wird auch von einer lokalen Evidenz gesprochen (Wright 2006), die die Wirksamkeit in einem bestimmten Setting beschreibt. Ein bekanntes Problem ist, dass Praxisprobleme meist sehr komplex als auch unscharf sind und wissenschaftliche Untersuchungen häufig sehr isoliert nur einen Aspekt tiefergehend beleuchten (Weibel et al. 2018). Für die Praxis ist am Ende entscheidend, ob die Evidenz nützlich ist. Forschungsansätze zum evidenzbasierten Management nehmen den Abgleich zwischen proklamiertem Wissen der Theorie und tatsächlicher Umsetzung in der Praxis vor (Barends et al. 2017; Rynes et al. 2002). Die Zielsetzungstheorie ist eine der empirisch am besten erforschten sowie bestätigten Theorien der Arbeits- und Organisationspsychologie (Kauffeld und Schermuly 2019). Kaum eine andere Motivationstheorie hat so viel Forschung angeregt und gleichzeitig Anwendung in der Praxis gefunden (Latham 2004; Locke und Latham 1990, 2013). Ziel des durchgeführten Abgleichs in der vorliegenden Studie ist es, ein fundiertes Bewusstsein für eine mögliche ForschungsPraxis-Lücke zur Zielsetzungstheorie zu schaffen. Folgenden Forschungsfragen soll nachgegangen werden: Wie sieht die Umsetzung des Führens mit Zielen in der betrieblichen Praxis aus? Welche Faktoren der Zielsetzungstheorie (Locke und Latham 1990) werden von Anwendern aktueller Zielsysteme wahrgenommen?

Das Wissen und der Umgang mit Zielpraktiken im Arbeitsalltag wurden durch Interviews mit Unternehmensvertretern erfasst, die bereits mit unterschiedlichen Zielsystemen in der betrieblichen Praxis gearbeitet haben. Ziel der Interviews war es Erfahrungen mit Zielsystemen in der Praxis zu eruieren sowie diese mit den Elementen der Zielsetzungstheorie abzugleichen. Die explorativen Interviews wurden mit 50 Vertretern aus einem Unternehmen der Automobilindustrie geführt. Diese Stichprobe bestand zu gleichen Anteilen aus Mitarbeitern sowie Personen mit Führungsverantwortung. Das Unternehmen besitzt mehrere Standorte in Deutschland sowie im Ausland und umfasst den gesamten Produktlebenszyklus von der Entwicklung bis 
zur Produktion. Die befragten Personen sind Vertreter aus unterschiedlichen Funktionen im Unternehmen. Insgesamt sind 16 Vertreter aus dem Produktionsumfeld, wie Montage oder Karosseriebau, 18 Vertreter aus dem produktionsnahen Umfeld, wie Instandhaltung oder Qualitätssicherung, und wiederum 16 Vertreter aus dem administrativen Bereich, wie Personalwesen oder Finanzwesen. Die Verteilung in den jeweiligen Tätigkeitsfeldern ist zwischen Führungskraft und Mitarbeiter ausgewogen. Bei der Auswahl der Untersuchungsteilnehmer war die Prämisse leitend, dass aus allen relevanten Unternehmensbereichen Interviewteilnehmer vertreten sind (Produktion, produktionsnaher und administrativer Bereich). Es sollten keine Aussagen einzelner Akteure untersucht werden, sondern das vorherrschende Wissen in der Gesamtorganisation, so wie es auch in der Praxis anzutreffen ist. In Bezug auf die Geschlechterverteilung ist ein größerer Anteil an männlichen Teilnehmern $(N=37)$ zu verzeichnen, der in der Automobilbranche üblich ist.

Die Gespräche wurden leitfadengestützt geführt, wobei es Möglichkeiten zum freien Explorieren gab. Der Interviewleitfaden umfasste die bisherigen Erfahrungen mit Zielsetzungen sowie Zielsystemen in der Organisation. Die berichteten Erfahrungen mit bisherigen Zielsystemen in der betrieblichen Praxis wurden anhand einer qualitativen Inhaltsanalyse angelehnt an die Grounded Theory Methode nach Glaser und Strauss (1967) analysiert. Die Auswertung der gewonnenen Daten erfolgte in zwei Stufen: über eine qualitative sowie eine quantitative Inhaltsanalyse. Hierbei entstand ein Kodiersystem in Form eines offenen Kodierens im Wechselverhältnis zwischen dem Gesprächsmaterial (induktiv) sowie der Theorie der Zielsetzung (Locke und Latham 1990; deduktiv). Bei der qualitativen Inhaltsanalyse wurden identifizierte Nennungen den übergeordneten Kategorien zugeordnet, um bestimmte Merkmalskombinationen gemeinsam abzubilden. Hierzu wurde neben der Definition der übergeordneten Kategorie auch Ankerbeispiele ermittelt. In einem zweiten Schritt erfolgte die Frequenzanalyse, indem Häufigkeiten der Kategoriennennungen ermittelt wurden, um die Intensitäten der Kategorien zu bestimmen. Die Nennungshäufigkeit ist ein Abbild der Zugänglichkeit einer Kategorie für die bewertende Person (Saracevic 2007). Dadurch entstanden numerische Werte, die Relationen und Häufigkeiten von äquivalenten Wahrnehmungen der Kategorien darstellen (Früh 2017). Durch die Intensität von bestimmten Kategorien wurden Leitmotive der interviewten Akteure identifiziert. Gleichzeitig konnten Themen mit geringerer Beachtung in der Praxis aufgezeigt werden, die gegebenenfalls zu kontraproduktiven Abwandlungen beim Einsatz von Zielen führen können. Die quantitativen Daten helfen die qualitativen Aussagen als zusätzliche Quelle der Relevanzeinschätzung zu kontrastieren (Lamnek 2008). Für die erste Forschungsfrage ,Wie sieht die Umsetzung des
Führens mit Zielen in der betrieblichen Praxis aus?" wurde sich ausschließlich auf die qualitative Analyse im Sinne der Exploration des Forschungsfeldes konzentriert. Die Erfahrungen wurden hier mit offenen Fragestellungen erfragt und zunächst nicht mit dem Zielsetzungsmodell abgeglichen. Für die zweite Forschungsfrage „,Welche Faktoren der Zielsetzungstheorie (Locke und Latham 1990) werden von Anwendern aktueller Zielsysteme wahrgenommen?" wurde sich sowohl der qualitativen Inhaltsanalyse als auch der genannten quantitativen Inhaltsanalyse bedient. Hierzu wurden die Aussagen mit den wissenschaftlichen Erkenntnissen von Locke und Latham (1990) verglichen. Dies erfolgte anhand eines Abgleichs mit den durch die Zielsetzungstheorie vorgegeben Kategorien sowie der Ermittlung numerischer Werte, die deskriptiv analysiert wurden.

\subsection{Wie sieht die Umsetzung des Führens mit Zielen in der betrieblichen Praxis aus?}

Um ein besseres Verständnis für die betrieblichen Bedingungen in der Automobilbranche zu erhalten, sollte im ersten Schritt eine Exploration stattfinden. Ziel war es zu ermitteln, wie die Umsetzung des Führens mit Zielen in der betrieblichen Praxis tatsächlich aussieht. Hierzu wurden die Interviewten gefragt, wie sie das Führen mit Zielen in der Organisation wahrnehmen und was hiervon für sie vorrangig im organisationalen Fokus steht. Basierend auf dieser offenen Exploration wurden vier übergeordnete Kategorien ermittelt, die den Einsatz von Zielen in dem Unternehmen beschreiben (siehe Tab. 1). Neben der ermittelten Kategorie ist eine Definition abgeleitet und Beispiele sind aufgezeigt.

Bei der Kategorie „Art der Ziele im Unternehmen“ konnte festgestellt werden, dass Ziele meistens nicht isoliert im Rahmen der individuellen Zielvereinbarung existieren, sondern sich in einem Gefüge mehrerer aufeinander aufbauender (zum Beispiel Verbesserung der Qualität zur Reduktion der Nacharbeit), aber auch konkurrierender Ziele befinden (zum Beispiel Einsparungen und Investitionen). Überwiegend werden Ziele in Form von Zielsetzungen anstatt in partizipativen Zielvereinbarungen festgelegt. Aufgrund der Komplexität in der Praxis werden Ziele auch in sogenannten Zielsystemen abgebildet, verfolgt und berichtet. Ein Zielsystem enthält Ziele aus mehreren Bereichen oder Teams. Die Befragten schilderten, dass es eine Vielzahl an Zielsystemen im Unternehmen gibt und diese oft parallel existieren. Beispiele dafür sind Ziele aus der Unternehmensstrategie, aus Kennzahlen-Dashboards, im Shopfloor Management, in der Teamarbeit sowie in der persönlichen Entwicklung. Diese in Einklang zu bringen ist laut Aussagen der Befragten eine der größten Herausforderungen. So sagte eine Führungskraft: „Die große Frage, die sich immer stellt: es gibt so viele Methoden und Ideen [um Ziele zu verfolgen]. Aber welche ist die Richtige für mich und welche 
Tab. 1 Ergebnisse der qualitativen Inhaltsanalyse zur Exploration des Untersuchungsfeldes

\begin{tabular}{|c|c|c|}
\hline Kategorie & Definition & Beispiel \\
\hline $\begin{array}{l}\text { (1 Art der Ziele im } \\
\text { Unternehmen }\end{array}$ & $\begin{array}{l}\text { Ziele versuchen die Komplexität } \\
\text { des Unternehmens abzubilden } \\
\text { und stehen immer in einem } \\
\text { Zusammenhang }\end{array}$ & $\begin{array}{l}\text { Unterschiedliche Quellen für Ziele } \\
\text { Zusammenhang zwischen Zielen (aufbauend oder konkurrierend) } \\
\text { Ziele im komplexen Gefüge der Organisation } \\
\text { Vielzahl an Zielsystematiken zur methodischen Abbildung von Zielen }\end{array}$ \\
\hline $\begin{array}{l}2 \text { Zweck von } \\
\text { Zielsystemen }\end{array}$ & $\begin{array}{l}\text { Gründe, warum mit Zielsystemen } \\
\text { in Unternehmen gearbeitet wird }\end{array}$ & $\begin{array}{l}\text { Betriebswirtschaftliche Steuerungsgründe } \\
\text { Motivationsgründe } \\
\text { Orientierung und einheitliche Ausrichtung }\end{array}$ \\
\hline $\begin{array}{l}3 \text { Zeitlicher } \\
\text { Horizont der } \\
\text { Zielsysteme }\end{array}$ & $\begin{array}{l}\text { Zeitraum auf den Ziele ausgelegt } \\
\text { sind im Unternehmen }\end{array}$ & $\begin{array}{l}\text { Kurzfristige vs. langfristige Ziele } \\
\text { Fokus auf operative Ziele } \\
\text { Integration von strategischen Zielen }\end{array}$ \\
\hline $\begin{array}{l}\text { (4) Art der } \\
\text { Zielmessung }\end{array}$ & $\begin{array}{l}\text { Vorgehensweisen zur Messung } \\
\text { der Zielerreichung }\end{array}$ & $\begin{array}{l}\text { Operationalisierung in Kennzahlen } \\
\text { Einführung von Ampellogiken (grün, gelb, rot) } \\
\text { Kontinuierliche Berichterstattung }\end{array}$ \\
\hline
\end{tabular}

können wir wirklich leben? Es werden zu viele diskutiert und angefangen“. In diesem Zusammenhang wurde von einem organisationalen Gedächtnis gesprochen, das folgende Aussage beinhaltet: „Es gab bereits mehrere Versuche ein neues Zielsystem einzuführen, das ist meistens nicht von langer Dauer".

Eine weitere Kategorie zeigt den „Zweck von Zielsystemen " in der Organisation. Grundsätzlich sind Zielsysteme sowohl aus Motivationsgründen als auch betriebswirtschaftlichen Steuerungsgründen eingeführt worden. Insbesondere der Einsatz von Zielen zum Steuern von Prozessen wurde von den Befragten vorrangig beschrieben. Demnach ist es ,ein wichtiges Element zur Steuerung der Fabrik“. Eine Führungskraft aus dem administrativen Bereich sagte: „Generell wurde mir klar, dass ohne diese Steuerung, wäre es viel schwieriger für uns dies alles unter ein Dach zu kriegen und das Boot in die richtige Richtung zu lenken, [...] Probleme abzustellen oder irgendwelche Potentiale zu nutzen“. Zielsysteme sollen auch Orientierung geben, wo sich das Unternehmen oder die Abteilung hin entwickeln soll. So sagte ein befragter Mitarbeiter, dass das genutzte Zielsystem „Orientierung gibt [...] in Sachen, was ist letztendlich wichtig und welche Prioritäten wir verfolgen sollen“. Die Herausforderung liegt nach den Aussagen im Interview in abgestimmten und aufeinander aufbauenden Zielen in einer komplexen sowie dynamischen Organisation. Dies soll mit aktuellen Zielsystemen vorrangig bewältigt werden. Daher wird auf den Zielsetzungsprozess fokussiert, um eine einheitliche Ausrichtung an gesamtunternehmerischen Zielen zu gewährleisten.

Der ,zeitlicher Horizont der Zielsysteme“ stellt eine weitere Kategorie dar, welche laut den Interviews sich überwiegend auf einen kurzfristigen Zeitraum bis zu maximal einem Jahr erstreckt. Übergeordnete Ziele werden jährlich vergeben und das Herunterbrechen auf Ziele für Abteilungen hängt von der Arbeit in den jeweiligen Abteilungen ab.
So sind in der Produktion eher kurzzyklische Ziele pro Tag oder sogar je Schicht beschrieben (zum Beispiel x Autos pro Tag/Schicht produzieren). Im Gegensatz hierzu sind die Ziele im Bereich der Entwicklung längerfristig ausgelegt (zum Beispiel x Erfindungsmeldungen im Jahr). Längerfristige strategische Ziele mit einem Zeithorizont länger als ein Jahr sind nur in einigen Fällen genannt worden. So äuBerte ein Mitarbeiter zu Zielen in aktuellen Zielsystemen im produktionsnahen Umfeld: „Für mich gibt es aktuell noch einen Nachteil [...], insbesondere für längerfristige Themen, die man nicht innerhalb eines Monats löst. Sie sind nicht abgebildet. Vielleicht muss man sich auf gewisse Sachen auch vorbereiten“. Eine ähnliche Sicht ergänzte eine Führungskraft aus dem Produktionsumfeld: „Es gibt dann auch ein paar Themen, wo wir [...] Maßnahmen definiert haben oder definieren mussten, die nicht zu einem kurzfristigen Erfolg führen und das ist noch so eine Problem in den Zielsystemen, dass man hier nochmal unterscheiden muss zwischen kurzfristig zu erreichende Ziele und langfristig zu erreichende Ziele“.

Dies schließt an die letzte Kategorie der „Zielmessung “ an, welche vorwiegend in Form von Kennzahlen erfolgt. Bedingt ist dies durch die Berichtspflicht zu bestimmten Stakeholdern im und außerhalb des Unternehmens. Hierzu wurden unterschiedliche Logiken entwickelt, um schnell $\mathrm{zu}$ sehen, ob das Ziel erreicht, teilweise erreicht oder verfehlt wurde. Da die Messung der Zielerreichung oft über Kennzahlen erfolgt, berichteten die Befragten, dass die Ziele selbst in Form von Kennzahlen operationalisiert sind. Um nicht regelmäßig neue Kennzahlen zu entwickeln, wird auf bereits im unternehmerischen Kontext vorhandene Kennzahlen zurückgegriffen. Diese sind jedoch nicht immer adäquat zu der Zielsetzung (zum Beispiel Mitarbeiterzufriedenheit über die Anzahl an durchgeführten Teamworkshops zu messen). Gleichzeitig wird von einem sehr hohen Aufwand für die Berichtserstattung berichtet und der Wunsch 
nach Konzentration auf die tatsächlich wichtigen Kennzahlen geäußert. So sagte ein Befragter: „Mein Wunsch ist zu sehen, was wir eigentlich an Kennzahlen haben und welche wir über Bord schmeißen, weil da fragt keiner mehr nach? [...] Und welche sind eigentlich effizient? Mit welchen steuere ich wirklich [...]?“ Grundsätzlich gibt es den Wunsch die Komplexität in der Praxis handhabbar zu machen und damit auch die Entscheidungsgrundlage zu verbessern. Ein Interviewteilnehmer äußerte seinen Wunsch sehr klar und deutlich: „Ich glaube, dass es sieben Kennzahlen sind, mit denen man ein Unternehmen steuern kann. Wir haben zu viele Kennzahlen. [...] Ich glaube aber auch, dass man mittelfristig nochmal definieren muss, was sind wirklich Kennzahlen eines Managements. [...] Das ist auch, wenn ich in einen Laden gehe, wo es 100 Paar Schuhe gibt, brauche ich verdammt lange mich zu entscheiden, welches Paar Schuhe ich kaufen will. Bei sieben ist die Auswahl wesentlich schneller. Das heißt, umso mehr ich zur Verfügung stelle, umso mehr verwickle ich mich auch, in der Entscheidung welche Kennzahl wichtig für mich ist. [...] Wenn ich jetzt alle Faktoren als eigene Kennzahl abbilde, verliere ich mich in einem Kennzahlspiel und nicht mehr in das was wir eigentlich machen sollen ,Autos bauen““.

In Bezug auf die Forschungsfrage „Wie sieht die Umsetzung des Führens mit Zielen in der betrieblichen Praxis aus? " kann grundsätzlich festgehalten werden, dass das Führen mit Zielen in dem Unternehmensbeispiel versucht die komplexen Zusammenhänge der Praxis abzubilden und anstatt mit individuellen Zielsetzungen eher mit Zielsystemen zu arbeiten. Hierdurch sind Ziele auf Team-, Organisations- oder Unternehmensebene im Fokus. Die Herausforderung hierbei ist die Vielzahl an existierenden Zielsystemen zu managen oder zu vereinfachen. Durch die Vielzahl an Zielsystemen werden unterschiedliche Schwerpunkte in der Zielarbeit gesetzt und für die Anwender ist es schwierig die wesentlichen Ziele zu identifizieren, was dysfunktionale Effekte, wie Überforderung, unterstützen kann. Das Unternehmen steht vor der Herausforderung den Mitarbeitern trotz der Komplexität im und außerhalb des Unternehmens eine klare Zielorientierung zu geben. Die Orientierung unterstützt die Ausrichtung des angestrebten Handelns und stellt somit eine wesentliche Funktion von Zielen dar. Hierdurch sollen sowohl motivationspsychologische Wirkungen erzielt, als auch eindeutig betriebswirtschaftliche Steuerungsgrößen berücksichtigt werden. Hierbei nimmt der Steuerungszweck einen größeren Anteil ein und erzeugt den Bedarf nach Berichterstattung auf unterschiedlichen Ebenen. Bezogen auf den zeitlichen Horizont der Zielsysteme sind neben kurz- und mittelfristigen Zielen (bis zu einem Jahr) auch langfristige Ziele (größer als ein Jahr) verstärkt zu kommunizieren, um auch die längerfristige Entwicklung im Auge zu behalten. Dies ist insbesondere für die zunehmend strategieorientierte Führungsarbeit im
Unternehmen von Relevanz. Die Art der Zielmessung sollte zu dem tatsächlich angestrebten Ziel passen. Ziele werden oft in Form von Kennzahlen gemessen. Obwohl es bereits viele unterschiedlichen Kennzahlen im Unternehmen gibt, sehen die Befragten noch Optimierungspotenzial bei der Auswahl und Definition von Kennzahlen. Zusammenfassend lässt sich sagen, dass das Führen mit Zielen eine hohe Relevanz in der Praxis darstellt und für unterschiedliche Zwecke sinnvoll ist. Herausforderungen werden vor allem in der Komplexität und der handhabbaren Messung von Zielen gesehen.

\subsection{Welche Faktoren der Zielsetzungstheorie (Locke und Latham 1990) werden von Anwendern aktueller Zielsysteme wahrgenommen?}

Nachdem die Situation zum Führen mit Zielen identifiziert und beschrieben wurde, sollte im Sinne des evidenzbasierten Managements analysiert werden, welche theoretischen Annahmen zur Zielsetzung sich in der Praxis wiederfinden. Hierzu wurde ein Abgleich der Faktoren der Zielsetzungstheorie (Locke und Latham 1990; siehe Abb. 1) mit den Wahrnehmungen der Anwendern aktueller Zielsysteme vorgenommen. Grundsätzlich konnten alle Variablen aus der Zielsetzungstheorie in der Praxis wiedergefunden werden, jedoch mit sehr unterschiedlicher Intensität. So wurde einerseits überprüft in wie vielen Interviews der Faktor vorkam und andererseits wie häufig er vorkam. Die angegebenen Häufigkeitsnennungen gelten in der Regel für Führungskräfte und Mitarbeiter in gleichem Maße. Falls es eine Abweichung zwischen den beiden Gruppen gab, wird dies zu dem jeweiligen Faktor entsprechend erwähnt. Grundsätzlich ist ein starker Fokus auf der Zielsetzung in der Praxis abzuleiten (410 Nennungen in 48 Interviews) und eine geringere Beachtung der Mediatoren sowie Moderatoren im Zielsetzungsprozess mit durchschnittlich $<62$ Nennungen pro Variable. Hierbei stellen die Rückmeldung (252 Nennungen in 45 Interviews), im Sinne eines kontinuierlichen Feedbackprozesses, und die Handlungsrichtung (116 Nennungen in 39 Interviews), im Sinne der Information zur Ausrichtung der Handlung auf einen Zielzustand, Ausnahmen mit deutlich höheren Nennungshäufigkeiten dar (siehe Abb. 2).

Insgesamt gibt es in fast allen Interviews knapp 410 Nennungen zur Art der Zielsetzung (zum Beispiel Vorgaben durch die Unternehmensführung, Ableitung aus der Bereichsstrategie, Ziele im persönlichen Gespräch). Bei diesem Faktor gab es einen Unterschied zwischen Führungskraft (248 Nennungen in 25 Interviews) und Mitarbeiter (162 Nennungen in 23 Interviews). Demnach haben Führungskräfte mehr mit Zielsetzungen zu tun als Mitarbeiter. Die explizite Verwendung von spezifischen (79 Nennungen in 29 Interviews) und schwierigen Zielen (31 Nennun- 


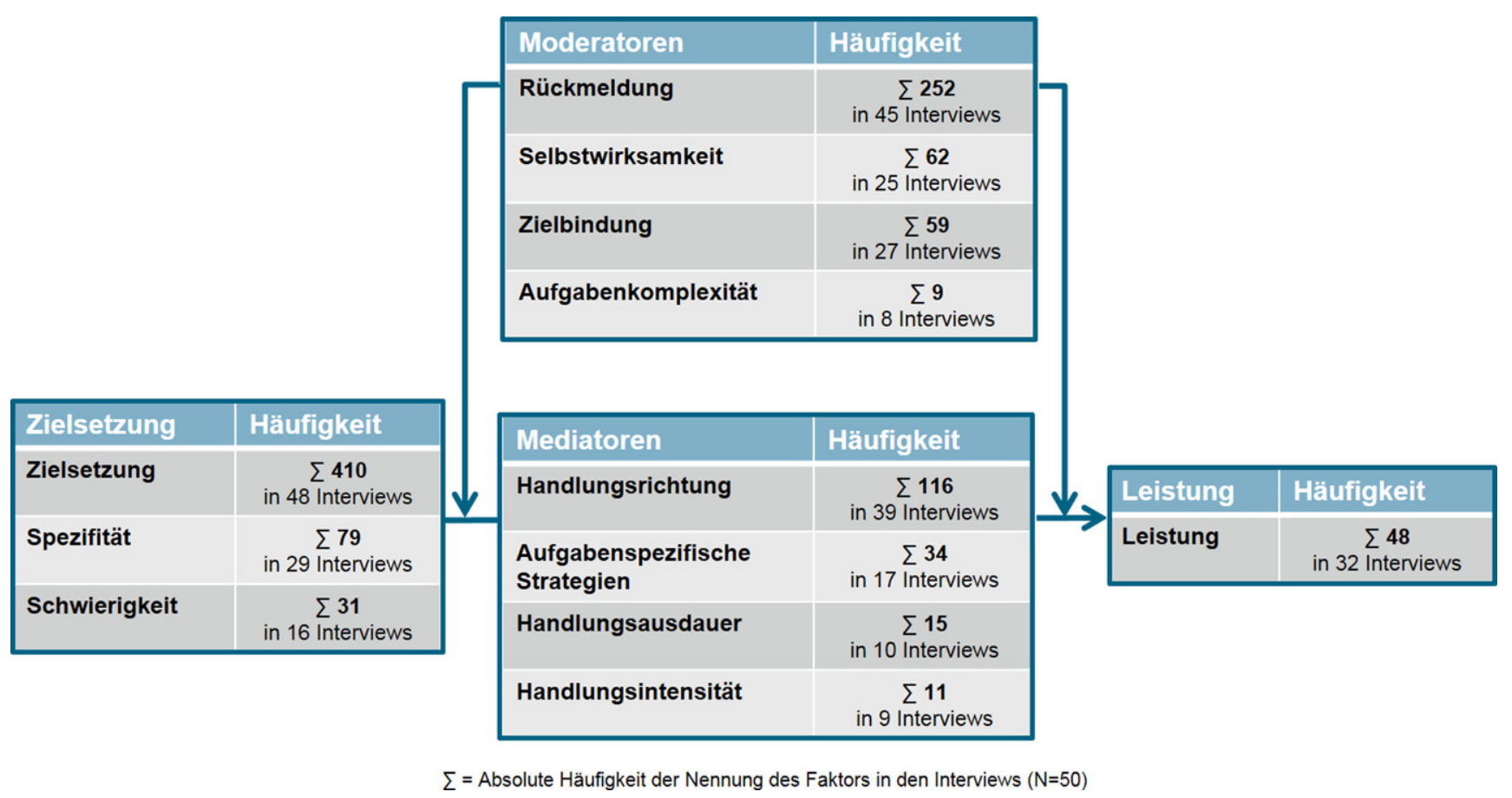

Abb. 2 Abgleich der Zielsetzungstheorie mit Interviewergebnissen. (Eigene Darstellung)

gen in 16 Interviews) wurde gering genannt. Die Ziele sind den Aussagen der Führungskräfte spezifischer und enthalten mehr Informationen zu Handlungsrichtung sowie -ausdauer (52 Nennungen in 20 Interviews zu 27 Nennungen in 9 Interviews bei den Mitarbeitern). Grundsätzlich werden Ziele in dem Unternehmensbeispiel einmal pro Jahr gesetzt und unterjährige Zielanpassungsprozesse finden trotz der immer volatileren Welt selten statt. Stattdessen wurden Priorisierungsprozesse genannt, wonach die gesetzten Ziele abhängig von den jeweiligen Einflussfaktoren eine höhere oder geringere Priorisierung erhalten. Generell ist den Interviewaussagen zu entnehmen, dass die Ziele oft in Form von Maßnahmen oder Aufgaben formuliert sind, die streng genommen Handlungen umfassen, um gesetzte Ziele zu erreichen (Hacker 1999; Hoch et al. 2009). So kann als Ziel ausgesprochen sein, dass die Fehlzeitenquote um $20 \%$ reduziert werden soll. Eine Maßnahme hierzu kann heißen gesundheitsförderliche Maßnahmen innerhalb des Betriebes anzubieten. Eine Vielzahl der Ziele sind in Form von Kennzahlen operationalisiert (zum Beispiel x Autos am Tag produzieren, Fehlerquote von $0 \%$ erreichen). Diese Kennzahlen stellen neben der zu erreichenden Zielgröße auch einen hilfreichen Indikator auf dem Weg der Zielerreichung dar und werden als Feedbackquelle genutzt. Eine Auswertung der Kennzahlen für Entscheidungsvorlagen stand hierbei nicht im Vordergrund. Vielmehr wurde in den Interviews betont, dass die Kennzahlen vorrangig zum Berichten und zur Dokumentation genutzt werden. Die fehlende Analyse von Kennzahlen für die Entscheidungsfindung, wurde be- reits in den Ausführungen von Pfeffer und Sutton (2007) als organisationale Schwachstelle beschrieben.

Nur knapp 50 Angaben beziehen sich auf die gezeigte Leistung in der Zielerreichung (zum Beispiel Messung der erreichten Ziele am Jahresende). Die Erfahrung zur Leistungsmessung wurde häufiger und von allen Führungskräften (31 Nennungen in 25 Interviews) genannt im Gegensatz zu Aussagen von nur einzelnen Mitarbeitern (27 Nennungen in 9 Interviews). Eine Erklärung ist, dass Führungskräfte in dem Unternehmen neben den Unternehmens- oder Abteilungszielen auch individuelle Zielvereinbarungen haben, auf dessen Basis ihr jährlicher Bonus berechnet wird. Hierdurch fließt die Zielerreichung direkt in die persönliche Bonusbewertung ein, was bei den Mitarbeitern so nicht gegeben ist.

Die Moderator- und Mediatorvariablen aus der Zielsetzungstheorie (siehe Abb. 1) sind in den Äußerungen der Interviewpartner seltener anzutreffen. Ein Faktor in der Zielarbeit wird jedoch hervorgehoben: der Einsatz von Feedback (Rückmeldung) zum Status der Zielbearbeitung (252 Nennungen in 45 Interviews). Hierbei spielt die Form des Feedbacks zunächst keine Rolle. So kann das Gespräch zwischen Führungskraft und Mitarbeiter oder im Team genauso hilfreich (120 Nennungen in 40 Interviews) sein wie die Information über Steuerungstafeln (132 Nennungen in 45 Interviews). Jedoch wird in der Verarbeitung des Feedbacks noch Optimierungspotenzial gesehen. Demnach sollte durch das Feedback ein Reflexionsprozess angeregt werden, um ein Lernen für zukünftige Zielhandlungen anzu- 
stoßen. Der Faktor der Zielbindung wird hingegen weniger beachtet als die wissenschaftliche Literatur empfiehlt (59 Nennungen in 27 Interviews). Angaben hierzu lauten, dass im Unternehmensbeispiel starre Rahmenbedingungen die Zielsetzung vorgeben und somit auch Vereinbarungen, die die Zielbindung stärken würden, weniger stattfinden. Somit sind vermehrt Zielvorgaben anzutreffen, die nach Aussagen der Befragten aufgrund fehlender Information und Kommunikation nicht immer nachvollziehbar sind. Dies ist in Bezug auf die Bindung und damit auch hartnäckigen Verfolgung der gesetzten Ziele kritisch zu sehen. Die Handlungsrichtung wurde mit bedeutsamen Abstand am zweithäufigsten genannt (116 Nennungen in 39 Interviews), hierunter fällt auch der Aspekt Orientierung durch Ziele. Neben der Richtung wurden wenig weitere Informationen für die Bearbeitung aus den Zielen abgeleitet, wie Handlungsausdauer (15 Nennungen in 10 Interviews) oder Handlungsintensität (11 Nennungen in 9 Interviews). Dies wiederum lässt den Rückschluss auf die Zielformulierung zu, dass sie nicht ausreichend Informationen enthält. In der Praxis wird dem Fehlen der Information entgegengewirkt, indem direkt Maßnahmen als Ziele gesetzt werden. Jedoch wird hierdurch das Handlungsrepertoire der Beteiligten nicht eigenständig weiter ausgebaut.

In Bezug auf die Frage „Welche Faktoren der Zielsetzungstheorie (Locke und Latham 1990) werden von Anwendern aktueller Zielsysteme wahrgenommen? " konnte identifiziert werden, dass Anwender von Zielsystemen im Unternehmensbeispiel die Faktoren unterschiedlich stark wahrnehmen. So werden einige Elemente sehr präsent (zum Beispiel kontinuierliches Feedback) und andere sehr gering (zum Beispiel Zielbindung, Zielspezifität, Handlungsausdauer und -intensität) wahrgenommen. Die unterschiedlich starke Wahrnehmung kann damit begründet sein, dass anstatt individueller Ziele eher Ziele auf organisationaler Ebene im Unternehmensbeispiel gesetzt werden. Damit sind individuumsbezogene Wirkmechanismen, wie die Zielbindung oder die Selbstwirksamkeit, nicht vorrangig im Betrachtungsfokus. Diese Faktoren sind insbesondere für die intrinsische Motivation wichtig, die einen wichtigen Einfluss auf die langfristige Leistungsfähigkeit der Mitarbeiter hat (Böhnisch et al. 2000). Eine stärkere Beachtung dieser Faktoren kann helfen den eingangs beschriebenen Vorwurf der vorwiegenden Konzentration auf die extrinsische Motivation beim Führen mit Zielen entgegenzuwirken. Weiterhin ist anzumerken, dass der Information, die von den Zielen ausgeht, wenig Bedeutung zugesprochen wird. Die hiermit verbundenen Faktoren, wie die Handlungsrichtung, -ausdauer, -intensität, werden bisher sehr gering wahrgenommen. Hierdurch wird ein wichtiger Wirkhebel bei der Zielsetzung zur Unterstützung der Orientierung im Unternehmen nicht ausreichend genutzt. Außerdem sind Ziele überwiegend für unternehmerische Steuerungszwecke ein- gesetzt, wodurch die geringe Beachtung der psychologischen Faktoren erklärt werden kann, die bereits von Bungard und Kohnke (2002) proklamiert wurde. Dieser Abgleich zeigt, dass in der Unternehmenspraxis bislang eher die Zielsetzungen per se als die damit verbundenen Faktoren der persönlichen Motivation wahrgenommen werden. Die vorhandenen wissenschaftlichen Erkenntnisse werden im Fallbeispiel der Automobilindustrie bei der Nutzung von Zielsystemen nicht konsequent angewendet.

\section{Impulse für Praxis und Forschung}

Bei der zusammenfassenden Betrachtung beider Forschungsfragen aus diesem Artikel, lässt sich festhalten, dass das Führen mit Zielen weiterhin eine hohe Relevanz in der unternehmerischen Praxis hat, jedoch der Einsatz zu betrieblichen Steuerungszwecken vorrangig ist. Im Folgenden werden die vier Kategorien aus der ersten Forschungsfrage (siehe 4.1) in Zusammenhang mit den Ergebnissen der Frequenzanalyse aus der zweiten Forschungsfrage (siehe 4.2) gebracht. Hieraus werden Impulse sowohl für die Praxis als auch für die weiterführende Forschung abgeleitet.

Die Art der Ziele im Unternehmen ist durch die komplexen und auch dynamischen Rahmenbedingungen im Unternehmen zu betrachten. So stehen Ziele immer im $\mathrm{Zu}-$ sammenhang zueinander und sind selten isoliert. Hierfür werden Ziele in Form von Zielsystemen im Unternehmen gesetzt, verfolgt und berichtet. Die größte Herausforderung ist das Abstimmen der Ziele zu einem übergeordneten Zielbild. Die intensive Wahrnehmung von Zielsetzungsprozessen (410 Nennungen in 48 Interviews) unterstreicht diese Herausforderung.

Der Zweck der Arbeit mit Zielen liegt vorrangig im Steuern von Prozessen und in der eingangs beschriebenen Herausforderung der Orientierung im Unternehmen. Organisationen nutzen ein abgestimmtes Zielbild vordringlich zur Orientierung und um dysfunktionalen Effekten, wie dem Silo-Denken entgegenzuwirken. Dies ist in der höheren Ausprägung der Nennungshäufigkeit der Handlungsrichtung zu erkennen (116 Nennungen in 39 Interviews). Die Orientierung könnte durch die verstärkte Beachtung der wissenschaftlichen Erkenntnisse um motivationale Effekte ergänzt werden. So kann sich auf die Stärkung der intrinsischen Motivation konzentriert werden, indem die Zielbindung (59 Nennungen in 27 Interviews) oder die Selbstwirksamkeit (62 Nennungen in 25 Interviews) der Mitarbeiter beim Zielsetzungsprozess mit beachtet wird. Hierdurch könnten weitere Dysfunktionen verringert werden, die durch eine zu starke Ausrichtung auf die extrinsische Motivation entstehen. Beispielhaft ist die $\mathrm{Zu}-$ nahme der Risikobereitschaft oder unethisches Verhalten zu nennen (Wegge 2015). Durch eine stärkere Einbindung 
der Mitarbeiter können realistische und umsetzbare Ziele identifiziert werden. Zeit- und Leistungsdruck (Hoppe und Rau 2017) kann wiederum durch die Konzentration auf das Wesentliche reduziert werden, weil so ein fokussierter Einsatz der eigenen Arbeitsleistung erfolgen kann. Durch eine Ziel- und Aufgabenklarheit kann die Befähigung der Mitarbeiter unterstützt und das Handlungsrepertoire der Beschäftigten erhöht werden. So ist auch eine Erklärung des Vorgehens und der Bedeutung von Zielen wichtig. So sagte eine Führungskraft: „,Was ist der Sinn und Zweck von dem Zielsystem? Warum wollen wir das gerne machen? Weil das sind ja alles mündige Mitarbeiter hier. Die möchten gerne abgeholt werden. Also das hilft einfach zu sagen im Vorfeld [...], was ist das und wo wollen wir hin“.

Zum zeitlichen Horizont der Zielsysteme ist $\mathrm{zu}$ sagen, dass in aktuellen Zielsystemen langfristige Ziele mit einem Zeithorizont über ein Jahr oft fehlen. Eine Erklärung hierfür kann sein, dass sich insbesondere auch Ziele in der Zukunft durch Rahmenbedingungen ändern können und diese oft noch sehr abstrakt sind. Dies sollte jedoch nicht davon abhalten, langfristige Ziele bereits frühzeitig in die Zielsysteme zu verankern und zusätzliche Zielanpassungsprozesse mit aufzunehmen. Durch diese können Ziele angepasst und auch zu gegebener Zeit weiter spezifiziert werden (Kauffeld und Schermuly 2019). Die Spezifizität von Zielen wurde von den Anwendern bisher eher gering wahrgenommen (79 Nennungen in 29 Interviews).

Die Art der Zielmessung sollte zu dem tatsächlich angestrebten Ziel passen und ein elementarer Bestandteil der Feedbackprozesse (252 Nennungen in 45 Interviews) sein. Somit könnte die Wirkung auf die tatsächlich gezeigte Leistung (49 Nennungen in 32 Interviews), auch für die Anwender der betrieblichen Zielsysteme wahrnehmbarer werden. Bei der Definition von Messgrößen kann die folgende Leitfrage unterstützen: „, Was kann ich wirklich mit meinem Handeln beeinflussen?". Oft sind die gesetzten Ziele, wie „eine effiziente Produktion zu erreichen“, sehr allgemein gefasst. Dabei sind Ziele im direkten Einflussgebiet, wie ,weniger Fehler beim Einbau von Teilen, um die Fälle der Nacharbeit zu reduzieren und somit die Effizienz in der Produktion zu steigern“, greifbarer. Diese Wirkzusammenhänge sind zu erklären, auch wenn sie in der Zielmessung nicht immer direkt abbildbar sind.

Zusammenfassend kann gesagt werden, dass die Zielsetzungstheorie immer noch ein gültiger Rahmen ist, der Anwendung in der betrieblichen Praxis findet und durch weitere Forschungen an die aktuellen Herausforderungen in den Unternehmen angepasst werden kann. Hieraus lassen sich Impulse sowohl für die Praxis als auch für die Forschung ableiten. Beginnend mit Impulse für die Praxis, sind die Folgenden zu nennen:
- Klarheit über den angestrebten Zweck beim Einsatz von Zielen - Der Zielsetzungsprozess ist von sehr hoher Relevanz in der Praxis, insbesondere zur betrieblichen Steuerung als auch um Orientierung zu geben. Daher sollte die Art der Zielsetzung bewusst gewählt werden. Zielvorgaben wirken schnell, sind zeiteffektiv und ermöglichen eine einheitliche Ausrichtung, wodurch sie im Unternehmenskontext ein adäquates Mittel darstellen und in den Interviews als vorrangiger Weg zur Zielsetzung genannt wurden. Sie haben allerdings auch ein Potential für dysfunktionale Effekte, wie Leistungsdruck oder Überforderung. Partizipative Zielvereinbarungen haben hingegen einen bindenden Charakter und sind insbesondere für die längere Zusammenarbeit geeignet, wenn bei Widerständen ausdauernd an einem Ziel gearbeitet werden soll. Wenn sich für Zielvorgaben entschieden wird, sollte der „,Tell-and-Sell“ Mechanismus (Latham et al. 1988) beachtet werden, um den Zweck und die organisatorische Wichtigkeit der Ziele zu erklären. Beides kann die Ausbildung der Zielbindung und somit auch den motivationalen Zweck von Zielsystemen in der Praxis unterstützen.

- Handlungsrepertoire erweitern - Die Ausbildung von handlungsspezifischen Strategien basierend auf den Zielen ist in den Interviews gering genannt worden. Die Beschreibung von Maßnahmen oder Handlungsanweisungen versuchen dies bereits im Zielsystem abzubilden. Jedoch wenn die Mitarbeiter mehr in die Verantwortung für die Ziele genommen werden und sie die Handlungen zur Zielerreichung selbstständig ableiten, wird sich ihr Handlungsrepertoire erweitern. Dies ist insbesondere für die Ausbildung von Selbstführungskompetenzen der Mitarbeiter wichtig (Nerdinger 2008), so dass Führungskräfte wieder Freiräume für strategische Themen erhalten.

- Nutzung von Kennzahlen - Die Verwendung von Kennzahlen hilft gemeinsame und nachvollziehbare Bewertungskriterien für die Zielerreichung festzulegen. Die Interviews zeigen, dass Kennzahlen den Abgleich zwischen Zielbearbeitung und -erreichung unterstützen, wenn sie adäquat zum jeweiligen Ziel gewählt werden. Bisher werden Kennzahlen kaum für die Entscheidungen von neuen Zielen herangezogen. Diese Rückkopplung sollte in zukünftigen Zielsystemen Beachtung finden.

Bezogen auf Impulse für die Forschung lassen sich Rückschlüsse auf die identifizierten Herausforderungen im Unternehmensbeispiel ziehen, wie die Orientierung für die $\mathrm{Ge}$ samtorganisation, Anpassungsprozesse in volatilen Zeiten oder die Nutzung von bereits erzeugten Fakten für weitere Entscheidungsprozesse. Zukünftige Forschungen sollten sich auf die Komplexität der betrieblichen Praxis konzentrieren, damit die gewonnenen Erkenntnisse auch Anwen- 
dung in der Praxis finden. Die folgenden Impulse lassen sich ableiten:

- Analysen auf Organisationsebene - Die Anwendung der Zielsetzungstheorie findet im Fallbeispiel vorwiegend auf Organisationsebene statt und verfolgt vorwiegend die Entwicklung eines abgestimmten Zielbildes zur Kommunikation der strategischen Ausrichtung des Unternehmens. Ziele können einen großen Mehrwert zur Kommunikation von strategischen Entscheidungen sowie zur Vermittlung von dessen Konsequenzen für die Arbeit bieten. Hierzu sollte sich auf das Wesentliche konzentriert werden und die Nachvollziehbarkeit sichergestellt sein. Dies kann durch Längsschnittuntersuchungen auf Organisations- und Gruppenlevel unterstützt werden, um die tatsächlichen Auswirkungen eines abgestimmten Zielbildes auf die Organisationsperformance zu überprüfen. Diese Erkenntnisse können der Praxis helfen längerfristig einer Zielsystematik treu zu bleiben, wenn die Wirksamkeit im Sinne einer lokalen Evidenz nachgewiesen ist (Wright 2006).

- Erweiterung der Theorie um Zielanpassungsprozesse - Immer klarer wird, dass jährliche Zielsetzungen in dynamischen und volatilen Zeiten der Transformation von Unternehmen nicht mehr ausreichen. Somit müssen Zielanpassungsprozesse in zukünftigen Zielsystemen mitgedacht werden. Forschungen zu Anpassungsprozessen, wie der Akkommodation oder Assimilation in Zielprozessen (Brandtstädter 2015), können die Zielsetzungstheorie für die aktuellen Rahmenbedingungen in Unternehmen weiterentwickeln. So können Anpassungsprozesse als Vermittler zwischen Feedback, unternehmerischen Rahmenbedingungen sowie Zielsetzung wirken.

- Vorgehensmodelle zur faktenbasierten Nutzung von Messgrößen - Zukünftige Forschungen zur Nutzung von Kennzahlen nicht nur zur Messung der Zielerreichung, sondern auch zur Entscheidung neuer Zielsetzungen, können den evidenzbasierten Umgang mit Zielsystemen stärken. Erkenntnisse hieraus sollen einen faktenbasierten Umgang mit Ergebnissen aus dem Zielsetzungsprozess bewirken. Es werden viele Fakten in den unternehmerischen Zielsystemen erzeugt, wie zum Beispiel durch die Erhebung von Kennzahlen. Diese sollten evidenzbasiert mit aussagefähigen Methoden ausgewertet und analysiert werden. Hier kann die Forschung insbesondere im Bereich der vorausschauenden Analyse (engl. ,predictve analytics") einen großen Mehrwert für die Praxis bieten (Mühlbauer 2017), indem Vorgehensmodelle, wie Big Data Analysen oder Ansätze von künstlicher Intelligenz, auch für den Bereich der Zielsetzung und nicht nur für das Berichtswesen mit überlegt werden.

\section{Kritische Reflexion und Ausblick}

Der methodische Zugang zu den eingangs beschriebenen Forschungsfragen wurde über Interviews mit Unternehmensvertretern aus verschiedenen Bereichen gewählt. Die reale Unternehmenspraxis sowie deren Komplexität können durch Interviews besser abgebildet werden als durch quantitative Fragebögen (Bogner et al. 2009). Gleichzeitig ist die Verallgemeinerbarkeit der Ergebnisse eingeschränkt. Generell wird bei qualitativen Methoden empfohlen offen an den Untersuchungsgegenstand heranzugehen. Ein komplett freies Vorgehen ohne Vorannahmen ist jedoch nach Brüsemeister (2008) nicht möglich. Somit bilden theoretische Vorannahmen bereits ein Gedankengerüst, welches allerdings in qualitativen Methoden offener als in quantitativen Methoden ist. Diese Kritik ist auch in der vorliegenden Untersuchung angebracht, da die Zielsetzungstheorie als zu untersuchende Theorie gegeben war. Jedoch wurde sie nicht deduktiv abgefragt, sondern Praktiker sollten über eigene Erfahrungen mit dem Führen durch Zielsetzungen und Zielsystemen berichten, um somit das Feld zu explorieren. Die Grounded Theory bietet die Möglichkeit, durch ein datenzentriertes und somit enges Arbeiten am Datenmaterial eine methodische Nachvollziehbarkeit zu schaffen und gleichzeitig ein hohes Abstraktionsniveau zu erreichen. Dadurch haben die Ergebnisse einen Praxisbezug und zeigen wichtige Anknüpfungspunkte, wo die Umsetzung der untersuchten Zielsetzungstheorie aktuell noch Schwächen in der Praxis hat. Jedoch können hiermit keine verallgemeinerbaren Aussagen getroffen werden, die für andere Unternehmen ebenso gelten. Die Untersuchung bezieht sich auf das untersuchte Beispiel in der Automobilindustrie, welches durch die tayloristische Entwicklung der Arbeitsorganisation bis heute noch geprägt ist (Ackermann 2013). In Branchen mit ausschließlich Wissensarbeitern wird diese Untersuchung sicherlich zu anderen Ergebnissen kommen, da andere Arbeitsformen und Arten von Zielsetzungen genutzt werden. Außerdem müsste für verallgemeinerbare Aussagen neben den quantitativen Angaben einer deskriptiven Frequenzanalyse noch eine statistische Absicherung durch interferenzstatistische Verfahren erfolgen. Ziel des durchgeführten Abgleichs war es ein fundiertes Bewusstsein für diese ForschungsPraxis-Lücke zu schaffen. Mit dem Vorgehen konnte überprüft werden, welche wissenschaftlichen Befunde den Weg in die Praxis in einem Automobilunternehmen gefunden haben und welche nicht. Zur Generalisierung der Erkenntnisse sind weiterführenden Untersuchungen in anderen Unternehmen und Branchen nötig. Zusätzlich wäre es von Interesse einen Gruppenvergleich zwischen unterschiedlichen Berufsgruppen vorzunehmen, wie zum Beispiel Personalern und Ingenieuren. Sie haben unterschiedlichen Biografien oder Hintergründe in der Ausbildung und es 
lässt sich vermuten, dass sie daher auch unterschiedliches Wissen zu Zielsetzungen im Arbeitsalltag anwenden.

Funding Open Access funding provided by Projekt DEAL.

Open Access Dieser Artikel wird unter der Creative Commons Namensnennung 4.0 International Lizenz veröffentlicht, welche die Nutzung, Vervielfältigung, Bearbeitung, Verbreitung und Wiedergabe in jeglichem Medium und Format erlaubt, sofern Sie den/die ursprünglichen Autor(en) und die Quelle ordnungsgemäß nennen, einen Link zur Creative Commons Lizenz beifügen und angeben, ob Änderungen vorgenommen wurden.

Die in diesem Artikel enthaltenen Bilder und sonstiges Drittmaterial unterliegen ebenfalls der genannten Creative Commons Lizenz, sofern sich aus der Abbildungslegende nichts anderes ergibt. Sofern das betreffende Material nicht unter der genannten Creative Commons Lizenz steht und die betreffende Handlung nicht nach gesetzlichen Vorschriften erlaubt ist, ist für die oben aufgeführten Weiterverwendungen des Materials die Einwilligung des jeweiligen Rechteinhabers einzuholen.

Weitere Details zur Lizenz entnehmen Sie bitte der Lizenzinformation auf http://creativecommons.org/licenses/by/4.0/deed.de.

\section{Literatur}

Ackermann, J. (2013). Lean Leadership - Eine situationstheoretische Untersuchung von erfolgsrelevantem Führungsverhalten in Veränderungsprozessen mit Lean-Ausrichtung am Beispiel eines Premiumherstellers der Automobilindustrie (Universität Köln, Hrsg.). Köln. https://kups.ub.uni-koeln.de/5153/1/Lean_ Leadership_Jana_Ackermann.pdf. Zugegriffen: 18. Juli 2018.

Bandura, A. (2012). Self-efficacy. The exercise of control (13. Aufl.). New York: Freeman.

Barends, E., Villanueva, J., Rousseau, D. M., Briner, R. B., Jepsen, D. M., Houghton, E., et al. (2017). Managerial attitudes and perceived barriers regarding evidence-based practice. An international survey. PloS one, 12(10), e184594. https://doi.org/10.1371/ journal.pone.0184594.

Barends, E., Janssen, B., \& Velghe, C. (2016). Rapid evidence assessment of the research literature on the effect of goal setting on workplace performance. Technical Report. https://www. cipd.co.uk/Images/rapid-evidence-assessment- of-the-researchliterature-on-the-effect-of-performance-appraisal-on-workplaceperformance_tcm18-16902.pdf. Zugegriffen: 30. Dez. 2017.

Bayer, U.C., Gollwitzer, P.M., \& Achtziger, A. (2010). Staying on track. Planned goal striving is protected from disruptive internal states. Journal of Experimental Social Psychology, 46(3), 505-514. https://doi.org/10.1016/j.jesp.2010.01.002.

Berson, Y., Halevy, N., Shamir, B., \& Erez, M. (2015). Leading from different psychological distances. A construal-level perspective on vision communication, goal setting, and follower motivation. The Leadership Quarterly, 26(2), 143-155. https://doi.org/10. 1016/j.leaqua.2014.07.011.

Biemann, T., Sliwka, D., \& Weckmüller, H. (2012). Auf gesicherte empirische Fakten setzen, statt auf Mythen vertrauen. PERSONALquarterly, 64(4), 10-17.

Bogner, A., Littig, B., \& Menz, W. (Hrsg.). (2009). Experteninterviews. Theorien, Methoden, Anwendungsfelder (3. Aufl.). Wiesbaden: VS.

Böhnisch, W., Freisler-Traub, A., \& Reber, G. (2000). Der Zusammenhang zwischen Zielvereinbarung, Motivation und Entgelt. Eine theoretische Analyse. Personal Zeitschrift für Human Resource Management, 1, 38-42.
Brandtstädter, J. (2015). Positive Entwicklung. Zur Psychologie gelingender Lebensführung (2. Aufl.). Berlin: Springer Spektrum. https://doi.org/10.1007/978-3-662-46946-0.

Breisig, T. (2001). Taylorismus. In H.-D. Zollondz (Hrsg.), Lexikon Qualitätsmanagement. Handbuch des modernen Managements auf der Basis des Qualitätsmanagements (S. 1142-1145). München: Oldenbourg.

Brüsemeister, T. (2008). Qualitative Forschung. Ein Überblick (2. Aufl.). Wiesbaden: VS. https://doi.org/10.1007/978-3-531-91182-3.

Bungard, W., \& Kohnke, O. (Hrsg.). (2002). Zielvereinbarungen erfolgreich umsetzen. Konzepte, Ideen und Praxisbeispiele auf Gruppen- und Organisationsebene (2. Aufl.). Wiesbaden: Gabler.

Burns, J. M. (1978). Leadership. New York: Harper \& Row.

Covey, S.R. (2018). Der 8. Weg. Mit Effektivität zu wahrer Größe (11. Aufl.). Offenbach: GABAL.

Domke, U., Granica, J. M., \& Hüter, M. (2019). Mutig führen. Wie Sie in Ihrem Unternehmen die Lust auf Verantwortung wecken. Stuttgart: Schäffer-Poeschel.

Drucker, P.F. (1954). The practice of management. New York: Harper \& Row.

Drucker, P.F. (2007). People and performance. The best of Peter Drucker on management. Boston: Harvard Business School Press.

Felfe, J. (Hrsg.). (2015). Trends der psychologischen Führungsforschung. Neue Konzepte, Methoden und Erkenntnisse. Göttingen: Hogrefe.

Franke, F., Ducki, A., \& Felfe, J. (2015). Gesundheitsförderliche Führung. In J. Felfe (Hrsg.), rends der psychologischen Führungsforschung. Neue Konzepte, Methoden und Erkenntnisse (S. 253-264). Göttingen: Hogrefe.

Früh, W. (2017). Inhaltsanalyse. Theorie und Praxis (9. Aufl.). UTB, Bd. 2501. Konstanz: UVK.

Glaser, B. G., \& Strauss, A. L. (1967). The discovery of grounded theory. Strategies for qualitative research. New York: Aldine de Gruyter.

Hacker, W. (1999). Zukunft gestalten? Probleme und Ergebnisse einer Probleme und Ergebnisse einer Psychologie zielgerichteten Handelns. In W. Hacker \& M. Rinck (Hrsg.), Zukunft gestalten. Bericht über den 41. Kongreß der DGfP in Dresden 1998. (S. 17-40). Lengerich: Pabst Science Publishers.

Haslam, S. A., Wegge, J., \& Postmes, T. (2009). Are we on a learning curve or a treadmill? The benefits of participative group goal setting become apparent as tasks become increasingly challenging over time. European Journal of Social Psychology, 39(3), 430-446. https://doi.org/10.1002/ejsp.546.

Hay Group (2011). Führungskräfte für eine neue Welt - Leadership Studie 2030. Was die Zukunft von Führungskräften verlangt. http://www.haygroup.com/downloads/de/Leadership_2030_ Whitepaper_DE_web.pdf. Zugegriffen: 7. Jan. 2018.

Heckhausen, H. (1977). Achievement motivation and its constructs. A cognitive model. Motivation and Emotion, 1(4), 283-329. https:// doi.org/10.1007/BF00992538.

Hoch, J.E., Wegge, J., \& Schmidt, K.-H. (2009). Führen mit Zielen. Report Psychologie, 34(7-8), 308-320.

Hoppe, J., \& Rau, R. (2017). Erlebte Beteiligung an der Zielsetzung. Zeitschrift für Arbeits- und Organisationspsychologie A\&O, 61(1), 18-30. https://doi.org/10.1026/0932-4089/a000230.

Kauffeld, S. (2019). Einführung in die Arbeits-, Organisations- und Personalpsychologie. In S. Kauffeld (Hrsg.), Arbeits-, Organisations- und Personalpsychologie für Bachelor. Mit 42 Tabellen 3. Aufl. Springer-Lehrbuch. (S. 1-20). Berlin: Springer.

Kauffeld, S., \& Schermuly, C.C. (2019). Arbeitszufriedenheit und Arbeitsmotivation. In S. Kauffeld (Hrsg.), Arbeits-, Organisationsund Personalpsychologie für Bachelor. Mit 42 Tabellen 3. Aufl. Springer-Lehrbuch. (S. 237-259). Berlin: Springer.

Klein, H.J., Molloy, J.C., \& Brinsfield, C.T. (2012). Reconceptualizing workplace commitment to redress a stretched construct. Revisiting assumptions and removing confounds. Academy of 
Management Review, 37(1), 130-151. https://doi.org/10.5465/ amr.2010.0018.

Lamnek, S. (2008). Qualitative Sozialforschung. Lehrbuch (4. Aufl.). Weinheim: Beltz.

Latham, G. P. (2004). The motivational benefits of goal-setting. Academy of Management Executive, 18(4), 126-129. https://doi.org/10. 5465/AME.2004.15268727.

Latham, G.P., \& Locke, E. A. (2007). New developments in and directions for goal-setting research. European Psychologist, 12(4), 290-300. https://doi.org/10.1027/1016-9040.12.4.290.

Latham, G.P., Erez, M., \& Locke, E. A. (1988). Resolving scientific disputes by the joint design of crucial experiments by the antagonists. Application to the Erez-Latham dispute regarding participation in goal setting. Journal of Applied Psychology, 73(4), 753-772. https://doi.org/10.1037/0021-9010.73.4.753.

Locke, E. A., \& Latham, G.P. (1990). A theory of goal setting \& task performance. Englewood Cliffs: Prentice-Hall.

Locke, E. A., \& Latham, G.P. (2002). Building a practically useful theory of goal setting and task motivation. A 35-year odyssey. American Psychologist, 57(9), 705-717. https://doi.org/10.1037/ 0003-066X.57.9.705.

Locke, E.A., \& Latham, G.P. (2006). New directions in goal-setting theory. Current Directions in Psychological Science, 15(5), 265-268. https://doi.org/10.1111/j.1467-8721.2006.00449.x.

Locke, E. A., \& Latham, G.P. (Hrsg.). (2013). New developments in goal setting and task performance. New York: Brunner-Routledge.

McClelland, D.C., Atkinson, J.W., Clark, R.A., \& Lowell, E.L. (1953). The achievement motive. New York: Appleton-CenturyCrofts.

Mühlbauer, D. (2017). People Analytics: Ein praxis-orientiertes Umsetzungsmodell. In ayway media (Hrsg.), Handbuch HR-Management. Human Resources, Digitalisierung, Human relations : New Work \& Arbeiten 4.0 : wie die Digitalisierung die Arbeitswelt verändert (1. Aufl. S. 269-279). Bonn: ayway media GmbH.

Nerdinger, F. W. (2008). Grundlagen des Verhaltens in Organisationen. Organisation und Führung, Bd. 2. Stuttgart: Kohlhammer.

Neubert, M.J. (1998). The value of feedback and goal setting over goal setting alone and potential moderators of this effect. A meta-analysis. Human Performance, 11(4), 321-335. https://doi.org/ 10.1207/s15327043hup1104_2.

Neuner, R., \& Neuner, S. (2014). Routinedaten effizient nutzen. Personalmagazin, 8, 38-40.

Pfeffer, J., \& Sutton, R.I. (2007). Hard facts, dangerous half-truths, and total nonsense. Profiting from evidence-based management. Boston: Harvard Business School Press.

Posch, A., \& Speckbacher, G. (2012). Führung in Familienunternehmen. Besonderheiten der Entscheidungsfindung und Verhaltenssteuerung und deren Auswirkung auf den Unternehmenserfolg. Zeitschrift für Betriebswirtschaft, 82(S3), 5-23. https://doi.org/ 10.1007/s11573-012-0566-z.

Putz, P., \& Lehner, J. M. (2002). Effekte zielorientierter Führungssysteme - Entwicklung und Validierung des Zielvereinbarungsbogens (ZVB). Zeitschrift für Arbeits- und Organisationspsychologie $A \& O, 46(1), 22-34$. https://doi.org/10.1026//0932-4089.46.1. 22.

Rousseau, D. M. (2006). Is there such A thing as "evidence-based management"? Academy of Management Review, 31(2), 256-269. https://doi.org/10.5465/AMR.2006.20208679.

Rynes, S.L., Colbert, A.E., \& Brown, K. G. (2002). HR Professionals' beliefs about effective human resource practices. Correspondence between research and practice. Human Resource Management, 4l(2), 149-174. https://doi.org/10.1002/hrm.10029.

Sackmann, S. A. (2017). Erfolgsfaktoren für neue Arbeitswelten - Unternehmenskultur und Führung. In B. Spieß \& N. Fabisch (Hrsg.), CSR und neue Arbeitswelten. Perspektivwechsel in Zeiten von
Nachhaltigkeit, Digitalisierung und Industrie 4.0 (ManagementReihe Corporate Social Responsibility (S. 375-385). Berlin: Springer Gabler.

Saracevic, T. (2007). Relevance. A review of the literature and a framework for thinking on the notion in information science. Part II: nature and manifestations of relevance. Journal of the American Society for Information Science and Technology, 58(13), 1915-1933. https://doi.org/10.1002/asi.20682.

Schwaab, M.-O. (Hrsg.). (2010). Führen mit Zielen. Wiesbaden: Springer.

Taylor, F.W. (1911). The principles of scientific management. New York: Harper \& Brothers.

Voss, R. (2015). Mit Zielen führen statt kontrollieren. https://www. hrweb.at/2015/05/fuehren-mit-zielen/. Zugegriffen: 11. Jan. 2019.

Wegge, J. (2015). Führen mit Zielen. In J. Felfe (Hrsg.), Trends der psychologischen Führungsforschung. Neue Konzepte, Methoden und Erkenntnisse (S. 179-190). Göttingen: Hogrefe.

Weibel, A., Schafheitle, S., \& Meidert, N. (2018). Evidenzbasiert entscheiden. Zeitschrift Führung Organisation, 87(03), 158-163.

Wright, M.T. (2006). Auf dem Weg zu einer theoriegeleiteten, evidenzbasierten, qualitätsgesicherten Primärprävention in Settings. In S. Heinrich \& M. Herrmann (Hrsg.), Prävention 1. Aufl. (Bd. 43, S. 55-73). Hamburg: Argument-Verl.

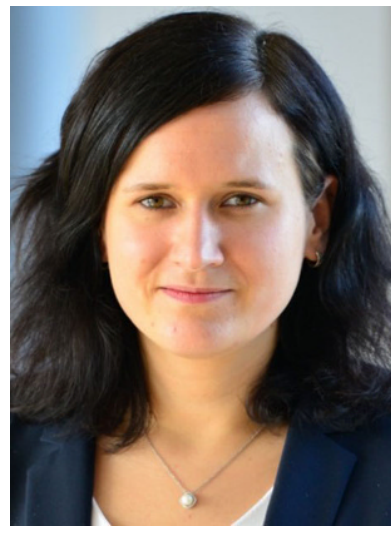

Dipl. Psych. Annika BurkhardtWaldherr ist Diplom Psychologin, promoviert in Kooperation mit einem Mobilitätsunternehmen und wird von Frau Prof. Dr. Kauffeld am Lehrstuhl für Arbeits-, Organisations- und Sozialpsychologie der Technischen Universität Braunschweig betreut. Ihr Schwerpunkt ist die Forschung zum evidenzbasierten Einsatz vom Führen mit Zielen in der Paxis.

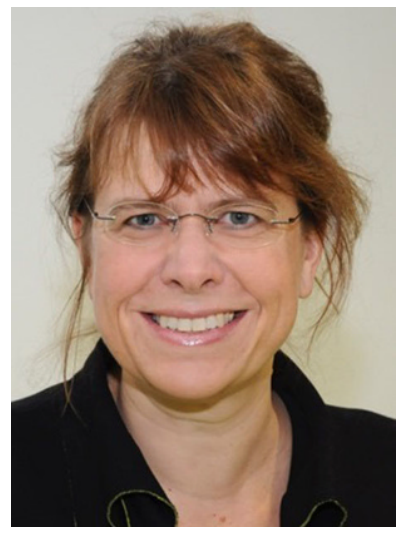

Prof. Dr. Simone Kauffeld ist Inhaberin des Lehrstuhls für Arbeit-, Organisations- und Sozialpsychologie der Technischen Universität Braunschweig. In ihrer Forschungstätigkeit setzt sie sich in zahlreichen Projekten mit den Themen Kompetenzentwicklung und -management (Training und Transfer), Team und Führung, Karriere/Coaching sowie Veränderungen in Organisation und Arbeit auseinander. Das Thema Digitalisierung ist als Querschnittsthema präsent. Als Herausgeberin hat sie die Zeitschriften „PersonalQUARTERLY“ und „Gruppe. Interaktion. Organisation" neu aufgesetzt und gibt Buchreihen zur Arbeits- und Organisationspsychologie heraus. Um aktiven Wissenstransfer zu leisten, hat sie 2008 die 4A-SIDE GmbH gegründet, die psychologische Expertise mit IT-Kompetenz verbindet. 